\title{
Predicting the risk of locoregional recurrence after early breast cancer: an external validation of the Dutch INFLUENCE-nomogram with clinical cancer registry data from Germany
}

\author{
Vinzenz Voelkel ${ }^{1,2}$ (1) $\cdot$ Teresa Draeger ${ }^{1,2} \cdot$ Catharina G. M. Groothuis-Oudshoorn ${ }^{2} \cdot$ Linda de Munck $^{4} \cdot$ Tom Hueting $^{3}$. \\ Michael Gerken ${ }^{1} \cdot$ Monika Klinkhammer-Schalke ${ }^{1} \cdot$ Miha Lavric $^{2} \cdot$ Sabine Siesling ${ }^{2,4}$
}

Received: 25 February 2019 / Accepted: 22 March 2019 / Published online: 29 March 2019

(C) The Author(s) 2019

\begin{abstract}
Purpose Follow-up after breast cancer treatment aims for an early detection of locoregional breast cancer recurrences (LRR) to improve the patients' outcome. By estimating individual's 5-year recurrence-risks, the Dutch INFLUENCE-nomogram can assist health professionals and patients in developing personalized risk-based follow-up pathways. The objective of this study is to validate the prediction tool on non-Dutch patients.

Material and methods Data for this external validation derive from a large clinical cancer registry in southern Germany, covering a population of 1.1 million. Patients with curative resection of early-stage breast cancer, diagnosed between 2000 and 2012, were included in the analysis $(n=6520)$. For each of them, an individual LRR-risk was estimated by the INFLUENCE-nomogram. Its predictive ability was tested by comparing estimated and observed LRR-probabilities using the Hosmer-Lemeshow goodness-of-fit test and C-statistics.

Results In the German validation-cohort, $2.8 \%$ of the patients developed an LRR within 5 years after primary surgery $(n=184)$. While the INFLUENCE-nomogram generally underestimates the actual LRR-risk of the German patients $(p<0.001)$, its discriminative ability is comparable to the one observed in the original Dutch modeling-cohort $(\mathrm{C}$-statistic German validation-cohort: 0.73, CI 0.69-0.77 vs. C-statistic Dutch modeling-cohort: 0.71, CI 0.69-0.73). Similar results were obtained in most of the subgroup analyses stratified by age, type of surgery and intrinsic biological subtypes.

Conclusion The outcomes of this external validation underline the generalizability of the INFLUENCE-nomogram beyond the Dutch population. The model performance could be enhanced in future by incorporating additional risk factors for LRR.
\end{abstract}

Keywords Mamma carcinoma $\cdot$ Personalized care $\cdot$ Follow-up $\cdot$ Tertiary prevention $\cdot$ Cancer registry $\cdot$ Health services research

Vinzenz Voelkel and Teresa Draeger equally contributed as first authors; Sabine Siesling and Miha Lavric equally contributed as senior authors.

Vinzenz Voelkel

vin.voelkel@posteo.de; v.h.f.voelkel@utwente.nl

1 Institute for Quality Control and Health Services Research, Tumor Center Regensburg/University of Regensburg, Regensburg, Germany

2 Department of Health Technology and Services Research, Technical Medical Centre, University of Twente, Enschede, The Netherlands

3 Evidencio, Medical Decision Support, Haaksbergen, The Netherlands

4 Department of Research, Netherlands Comprehensive Cancer Organisation (IKNL), Utrecht, The Netherlands

\section{Purpose}

Breast cancer is the most frequent malignancy among the female population. Worldwide, approximately 1.7 million women per year are diagnosed with this kind of tumor (Stewart and Wild 2014). Due to early detection, leading to lower stage at diagnosis, better treatment strategies and a wider awareness for the disease in general, the survival rates of breast cancer patients have been increasing considerably during the past decades (Yoshimura et al. 2018; Holleczek et al. 2011; Holleczek and Brenner 2012). In early-stage breast cancer, radical removal of the tumor is the first choice of treatment, which is often followed by adjuvant radiation and/or systemic therapy. Additionally, tertiary prevention is of great importance. Patients undergo regular follow-up 
visits to detect possible locoregional recurrences (LRR) in an early stage. Thus, subsequent distant metastases with a high risk of poor outcome shall be avoided (Lu et al. 2009; Sangen et al. 2013). Fortunately, the LRR-rate is generally low. Only $4 \%$ of all Dutch breast cancer patients from the year 2003 were diagnosed with a local recurrence event within 10 years after primary surgery, and only $2 \%$ developed a regional recurrence (Geurts et al. 2017). Moreover, the risk of LRR is not the same for every patient and changes over time. Since every follow-up visit potentially affects a patient's quality of life (Puglisi et al. 2014) and is a burden on health care facilities, it might be reasonable to personalize follow-up schemes by focusing on high-risk patients. In 2015, Witteveen et al. developed a "prognostic nomogram for the estimation of annual risk of locoregional recurrence in early breast cancer patients" (Witteveen et al. 2015). This so-called INFLUENCE-nomogram is based on over 37,000 patients of the Netherlands cancer registry (NCR) from the years 2003-2006. After entering several patient, tumor, and treatment characteristics (age, tumor size, nodal involvement, grade, ER-/PR-status, multifocality, radiotherapy, chemotherapy, and endocrine therapy), it estimates the individual risk of developing a recurrence within the first 5 years after surgery, as well as conditional annual risks based on multivariable logistic regression models (Witteveen 2019; Witteveen 2015). To assess the validity of this online tool, it was tested successfully on another Dutch cohort of more than 12,000 patients from the years 2007-2008 (Witteveen et al. 2015). However, until today it is unclear whether the nomogram is generalizable to foreign populations and health care systems, which would contribute to demonstrate its clinical relevance. This study aims to test the external validity of the INFLUENCE-nomogram on a representative cohort from a large German clinical cancer registry with additional emphasis on important patient subgroups.

\section{Methods}

Data for this external validation derive from a clinical cancer registry (Tumor Center Regensburg/University of Regensburg, Institute for Quality Control and Health Services Research (Tumorzentrum Regensburg 2019), which systematically collects medical records of all tumor patients registered within a large political district in the south of Germany compromising approximately 1.1 million inhabitants (Bayerisches Landesamt für Statistik und Datenverarbeitung 2014). Information on each patient includes demographics, tumor characteristics, surgical procedure, and adjuvant treatment (Table 1). Over 50 hospitals and 1500 registered health professionals immediately report newly diagnosed recurrent events. To obtain actual information on vital status, a regular exchange with local registration and public health offices takes place. All following analyses are performed in compliance with German data protection laws.

To be included in the validation process, patients had to fulfill certain inclusion criteria dictated by the INFLUENCE-nomogram. All patients with a histologically confirmed primary invasive breast cancer diagnosis (ICD10-GM C50 (DIMDI 2017) between 2000 and 2012, who according to their OPS-code (Institut 2019) received curative R0-resection (Hermanek and Wittekind 1994) were eligible for inclusion. Patients who received neoadjuvant treatment were diagnosed with distant metastasis or T4-tumors were excluded for analysis. Of all patients meeting the inclusion criteria, only patients without missing data on any relevant item and a follow-up time of at least 5 years were used for validation of the 5 -year overall risk predictions. To assess the separate predictions for the five conditional annual risks, patients were required to have a minimum follow-up time of $1,2,3,4$ and 5 years, respectively. To account for selection bias due to exclusion, a sensitivity analysis comparing LRRrates was performed.

Patient, tumor and treatment characteristics of the German validation-cohort were compared to those of the Dutch cohort from 2003 to 2006, on which the prediction tool was originally built (hereafter referred to as "modelingcohort") (Witteveen et al. 2015). For this purpose, it was refrained from using $\chi$-square tests, since with large numbers of observation units, they are overly sensitive to minor, and from a clinical point of view irrelevant, differences in distribution.

To obtain the German validation-cohort's interval-specific LRR-rates, the life-table method based on five annual observation periods was employed. Thereafter each patient's individual recurrence risk was estimated using the algorithm behind the INFLUENCE-nomogram (obtained from the online medical prediction platform Evidencio, www.evide ncio.com (Witteveen 2019) both for the whole 5-year postoperative period and for every year separately. To assess general prediction accuracy on the overall 5-year LRRrisk, the Hosmer-Lemeshow goodness-of-fit test based on quintiles was employed (Hosmer et al. 2013). Confidence intervals for the observed LRR-rates were obtained using Clopper-Pearson's exact method based on binomial distribution (Clopper and Pearson 1934). Moreover, a calibration chart was plotted to visualize the correlation between all predicted probabilities and the observed primary LRRrates. Due to a low annual number of primary LRRs in the validation-cohort, it was refrained from performing separate Hosmer-Lemeshow tests for every year.

The ultimate aim of prediction tools like the INFLUENCE-nomogram is to discern between high- and low-risk patients (Steyerberg et al. 2010). This discrimination-ability can be evaluated using the $\mathrm{C}$-statistic/ area under the curve (AUC) of the receiver-operator characteristic (ROC) 
Table 1 A patient and tumor characteristics; B treatment characteristics
Validation-cohort Germany (2000-2012) $n=6520$

\% $\%^{*}$

(A) Patients and tumor characteristics

Age category at diagnosis (years)

$<50$

$50-59$

$60-69$

$\geq 70$

Histologic type

Ductal

Lobular

Mixed

Other

Grading

1

2

3

Unknown

Tumor size (mm)

$<20$

$20-50$

$>50$

Unknown

Multifocal

No

Yes

Unknown

Lymph node status

Negative

1-3 positive

$>3$ positive

Unknown

ER status

Negative

Positive

Unknown

PR status

Negative

Positive

Unknown

Her2neu status ${ }^{+}$

Negative

Positive

Unknown

Intrinsic biological subtyp

Luminal A/B

Her2neu positive

Triple negative

Unknown
1408

1579

1866

1667

21.6

24.2

28.6

25.6

73.8

12.4

4.9

8.8

15.3

56.9

27.8

1812

n.a.

3699

2572

249

n.a.

5432

1088

n.a.

4660

1079

781

n.a.

982

5538

n.a.

56.7

39.4

3.8

83.3

16.7

71.5

16.5

12.0

15.1

84.9

1487

5033

n.a.

22.8

77.2

4850

1074

596

5661

249

521

89

81.9

18.1

88.0

3.9

8.1
Modeling-cohort Netherlands (2003-2006)

$n=37,278$

n \%*

9779

26.2

$10,601 \quad 28.4$

$8421 \quad 22.6$

$8477 \quad 22.7$

$29,582 \quad 79.4$

$4000 \quad 10.7$

$1552 \quad 4.2$

$2144 \quad 5.8$

$7628 \quad 22.0$

$15,595 \quad 44.9$

$11,479 \quad 33.1$

2576

$22,611 \quad 61.2$

$13,243 \quad 35.8$

$1094 \quad 3.0$

330

$23,237 \quad 84.8$

$4168 \quad 15.2$

9873

$22,516 \quad 61.3$

$10,093 \quad 27.5$

$4119 \quad 11.2$

550

5417

18.8

$23,433 \quad 81.2$

8428

9580

33.7

18,877

66.3

8821

13,832

85.2

2405

14.8

21,041

n.a. .5

15.2 
Table 1 (continued)

\begin{tabular}{|c|c|c|c|c|}
\hline & \multicolumn{2}{|c|}{$\begin{array}{l}\text { Validation-cohort Germany (2000-2012) } \\
n=6520\end{array}$} & \multicolumn{2}{|c|}{$\begin{array}{l}\text { Modeling-cohort Netherlands } \\
(2003-2006) \\
n=37,278\end{array}$} \\
\hline & $n$ & $\% *$ & $n$ & $\% *$ \\
\hline \multicolumn{5}{|c|}{ (B) Treatment characteristics } \\
\hline \multicolumn{5}{|l|}{ Type of surgery } \\
\hline Breast conserving & 4695 & 72.0 & 21,049 & 56.5 \\
\hline Mastectomy & 1722 & 26.4 & 16,229 & 43.5 \\
\hline Unknown & 103 & & n.a. & \\
\hline \multicolumn{5}{|l|}{ Chemotherapy } \\
\hline No & 3518 & 54.0 & 23,886 & 64.1 \\
\hline Yes & 3002 & 46.0 & 13,392 & 35.9 \\
\hline \multicolumn{5}{|l|}{ Radiotherapy } \\
\hline No & 1573 & 24.1 & 12,783 & 34.3 \\
\hline Yes & 4947 & 75.9 & 24,495 & 65.7 \\
\hline \multicolumn{5}{|l|}{ Endocrine therapy } \\
\hline No & 1501 & 23.0 & 21,696 & 58.2 \\
\hline Yes & 5019 & 77.0 & 15,582 & 41.8 \\
\hline
\end{tabular}

(Bamber 1975). A C-statistic (AUC) of 1.0 indicates perfect predictive ability, whereas 0.5 represents no predictive discrimination. The corresponding confidence intervals were obtained by DeLong's method (DeLong et al. 1988). Based on the ROC curve of the German validation-cohort, the model's joint-optimum for sensitivity and specificity based on an "ideal" cutoff risk value was calculated using Youden's J-statistic (Youden 1950). With nonparametric estimation, ROC actually requires fewer distributional assumptions than the Hosmer-Lemeshow test (Youngstrom 2013). Thus, it is not only possible to validate the discrimination-ability of the 5-year overall predictions but also of the time-dependent model estimating annual risks (conditional on the fact that a patient did not develop a recurrence in the previous year). Additionally to that, analyses stratified by age, type of surgery, and intrinsic biological subgroups (following the definition of the 12th St. Gallen International Breast Cancer Conference, 2011 (Goldhirsch et al. 2011) could be performed.

All significance tests were two-sided with a significance level of 0.05 and results are displayed with $95 \%$ confidence intervals (CI). The findings of this survey are presented in strict compliance with the Strengthening the Reporting of Observational studies in Epidemiology (STROBE) statement (Elm et al. 2007). During this study, IBM SPSS 25 (IBM Corp., SPSS for Windows, Armonk, NY, USA), as well as $\mathrm{R}$ version 3.3.2 (R Foundation for Statistical Computing, Vienna, Austria; https://www.R-project.org/) and the R packages "predictABEL" (Kundu et al. 2011), "Hmisc"
(www.CRAN.R-project.org/package=Hmisc) and "pROC" (Robin et al. 2011) were used.

\section{Results}

Within the observed German political district, 8398 patients diagnosed with invasive breast cancer between 2000 and 2012 fulfilled all inclusion criteria dictated by the INFLUENCE-nomogram (no distant metastases or T4-stage at time of surgery, no neoadjuvant therapy, no micro- or macroscopically incomplete surgery). Of them, 1878 (22.4\%) had to be excluded due to missing data on UICC TNM, grading, hormone receptor or lymph node status, leaving 6520 patients that could be used for validation (Fig. 1).

The median (mean) follow-up time calculated over the whole validation-cohort was 8.5 (8.6) years (using Korn's Kaplan-Meier potential follow-up method (Schemper and Smith 1996). One hundred and eighty-four patients developed an LRR within 5 years, which is equivalent to an overall 5-year LRR-rate of $2.8 \%$. This percentage is not significantly different to the LRR-rate observed in the Dutch modeling-cohort $(2.6 \%, p=0.205)$. The LRR-rate among the 1878 excluded patients was $2.9 \%$, which, according to the sensitivity analysis, is not significantly different from the included patients' LRR-rate $(p=0.902)$. Thus, selection bias due to exclusion seems unlikely.

The observed LRR-rates in the German validationcohort range between $0.4 \%$ and $0.8 \%$ per year (Fig. 2a). After stratifying for intrinsic biological subtypes, a similar 
Fig. 1 Flowchart of study patient selection

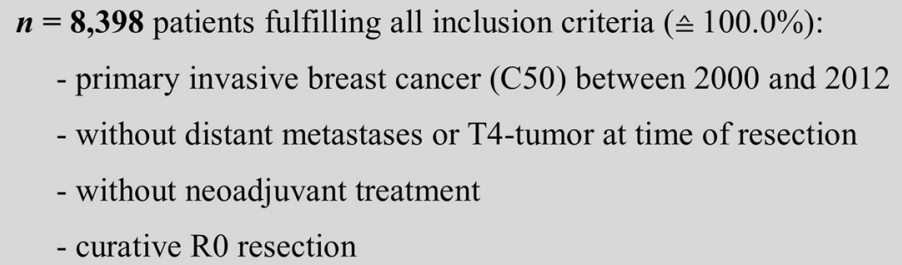

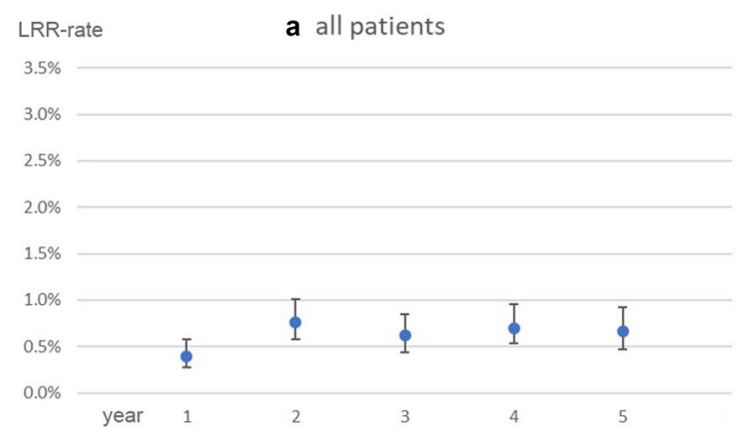

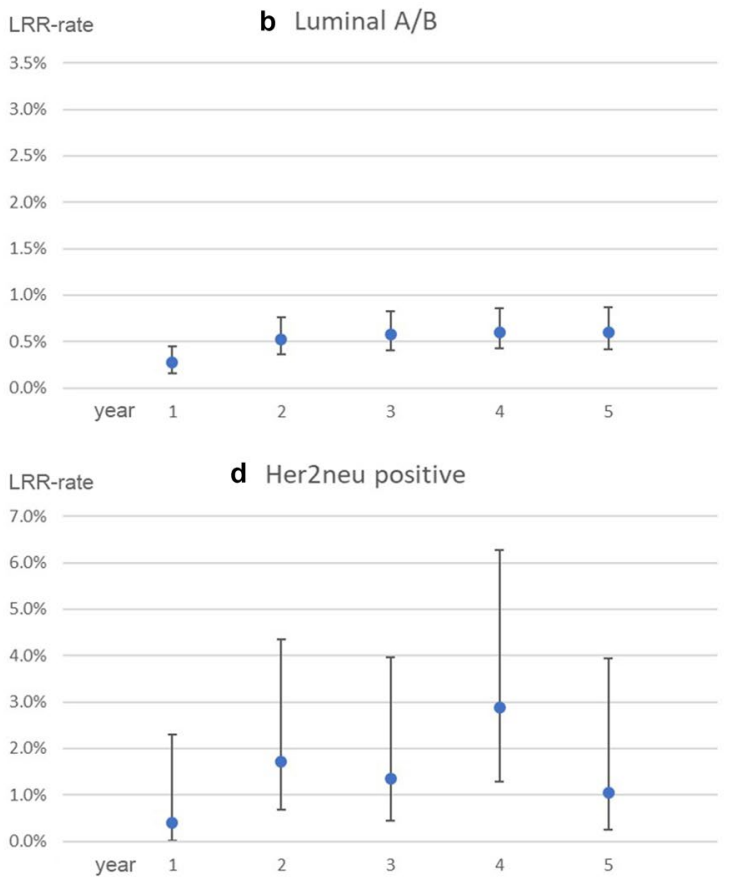

Fig. 2 Annual risks for LRR observed in the validation-cohort with 95\% CI. a All patients. b Luminal A/B. c Triple negative. d Her2neu positive

trend can be seen among Luminal A/B patients (Fig. 2b). Triple-negative patients show an incidence-peak in the second year after surgery (LRR-rate year 2: 2.9\%, Fig. 2c). With Her2neu-positive patients, the LRR-rate reaches its maximum in year 4 (LRR-rate year 4: $3.0 \%$ ), although no clear trend can be identified (Fig. 2d). However, this subgroup is formed by 249 patients, which goes along with a small number of LRR-events. Consequently, the confidence intervals for the Her2neu patients' LRR-rate are quite large (e.g. year 4: CI 1.4\%-6.4\%).
The German validation- and the Dutch modeling-cohort exhibit highly comparable patient and tumor features. Generally, there are low absolute differences in relative proportions of variables, e.g. age category at diagnosis, tumor size, or estrogen receptor (ER)-status (Table 1a). More substantial discrepancies between the Dutch and the German patients can be observed among the treatment variables (Table $1 \mathrm{~b}$ ). While $72 \%$ of the German patients received breast-conserving surgery, just $57 \%$ of the Dutch patients were treated this way. Additionally, there was a higher rate of endocrine 
therapy to be observed in the German cohort, although hormone status did not differ substantially between groups. Adjuvant chemo- or radiotherapy was performed more often in Germany, too.

\section{Validation}

Initially, the 5-year LRR-risks estimated by the INFLUENCE-nomogram were compared to the actually observed recurrence rates within the validation-cohort using the Hosmer-Lemeshow goodness-of-fit test, which returned a $p$ value lower than 0.001 , indicating poor accuracy. However, looking at the calibration chart it becomes obvious that the absolute differences between observed and predicted risks are only moderate and come along with relatively large confidence intervals (Fig. 3a). Notwithstanding that, the INFLUENCE-nomogram tends to underestimate the 5-year LRRrisk. In the quintile comprising the patients with the lowest risk estimations, a mean predicted LRR-risk of $0.5 \%$ stands against a mean observed LRR-rate of $0.9 \%$ (CI $0.5-1.6 \%$ ). In the highest risk quintile, the difference between the mean predicted LRR-risk and the mean observed LRR-rate is even significant (mean predicted: $5.6 \%$ vs. mean observed: $9.4 \%$, CI 7.7-11.3\%).

The INFLUENCE-nomogram's discrimination-ability was evaluated by computing the C-statistic/area under

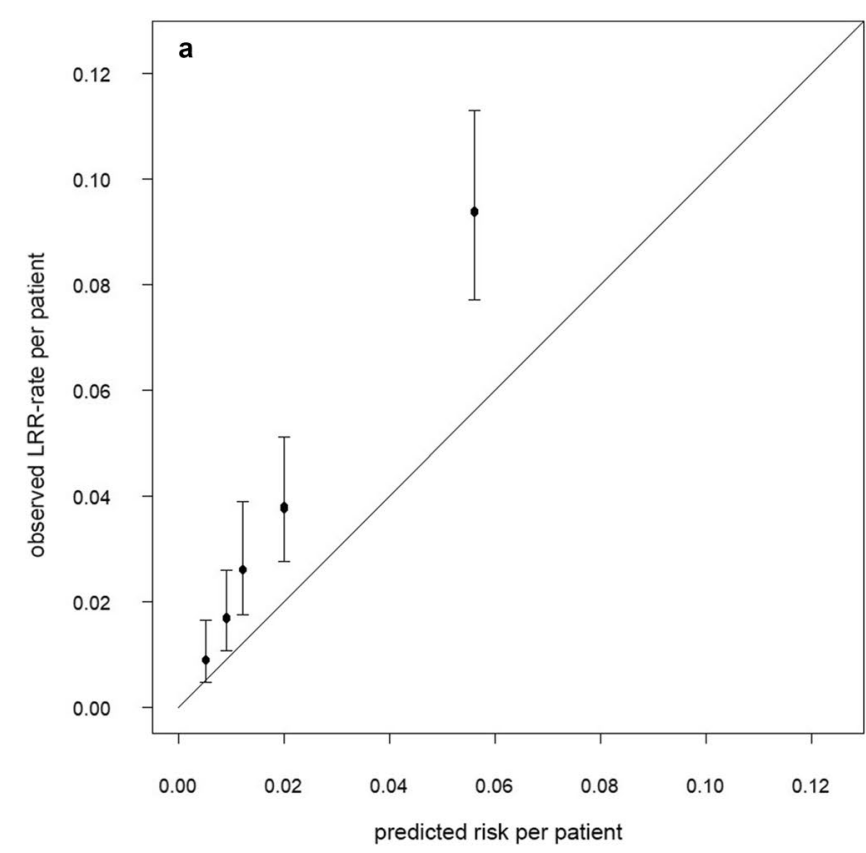

Fig. 3 5-year overall LRR-risk. a Calibration chart of the validationcohort based on quintiles. Hosmer-Lemeshow goodness-of-fit test: $p<0.001$. b 5-year overall LRR-risk. ROC curve of the validation- the ROC curve, which was 0.73 (CI 0.69-0.77), indicating reasonably good performance (Fig. 3b). Given the according to Youden's J-statistic ideal threshold of 1.6\%, the joint-maximum of sensitivity and specificity is $70.7 \%$ and $65.0 \%$, respectively. The discrimination-ability after predicting risks for each year separately is decreasing over time. While the C-statistic (AUC) is 0.78 (CI 0.66-0.90) for year 1 and 0.73 (CI $0.65-0.80$ ) for year 2, it decreases to 0.50 (CI $0.41-0.60)$ in year 5 , meaning there is no discriminative ability left (Fig. 4a-e).

The discriminative ability based on LRR-risk estimations for the whole 5-year period was tested for each of the age categories separately. The C-statistic (AUC) varied between 0.69 (CI $0.61-0.78$ ) for patients younger than 50 years and 0.75 (CI $0.68-0.82$ ) for patients older than 70 years (Table 2 ). Therefore, age is not an effect modifier for model performance. The same is true for the type of surgery (Table 2): the C-statistic is almost identical for patients with breast-conserving therapy (C-statistic/AUC 0.72, CI 0.67-0.78) and mastectomy (C-statistic/AUC 0.72 , CI $0.65-0.78$ ). Finally, another stratified analysis was performed for intrinsic biological subtypes (Table 2). For luminal A/B-tumors and triple-negative tumors, the C-statistic/AUC was 0.71 (CI 0.66-0.76) and 0.73 (CI 0.64-0.82), respectively. For Her2neu-positive patients, the model performed considerably worse (C-statistic/AUC 0.60 , CI 0.43-0.76).

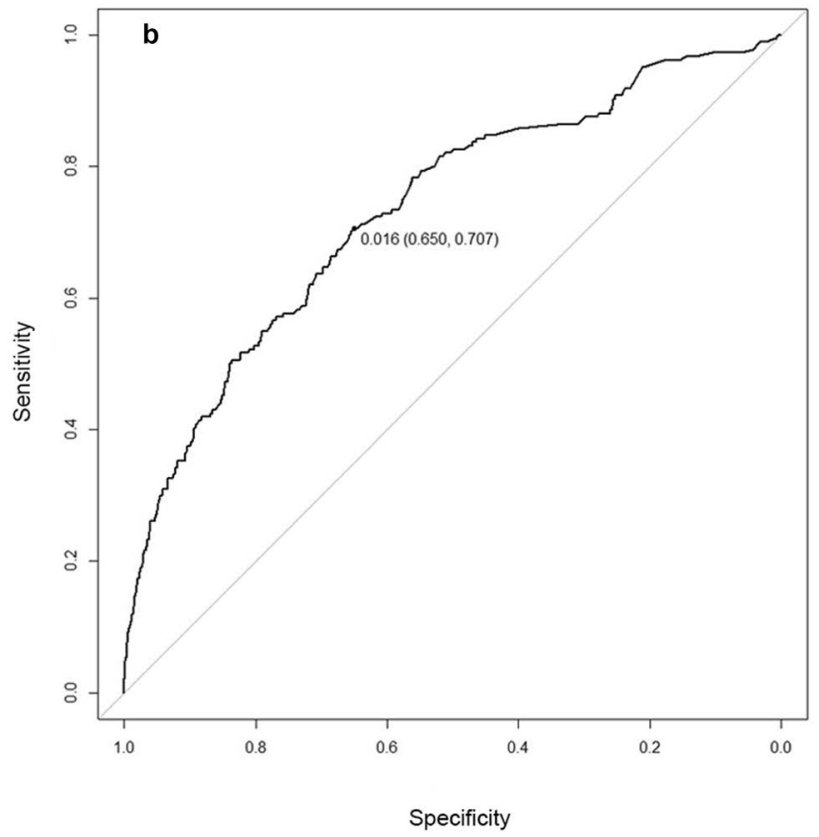

cohort (C-statistic/AUC: 0.73, CI 0.69-0.77) together with the optimal discrimination-threshold according to Youden's J-statistic: cutoff value (specificity, sensitivity) 

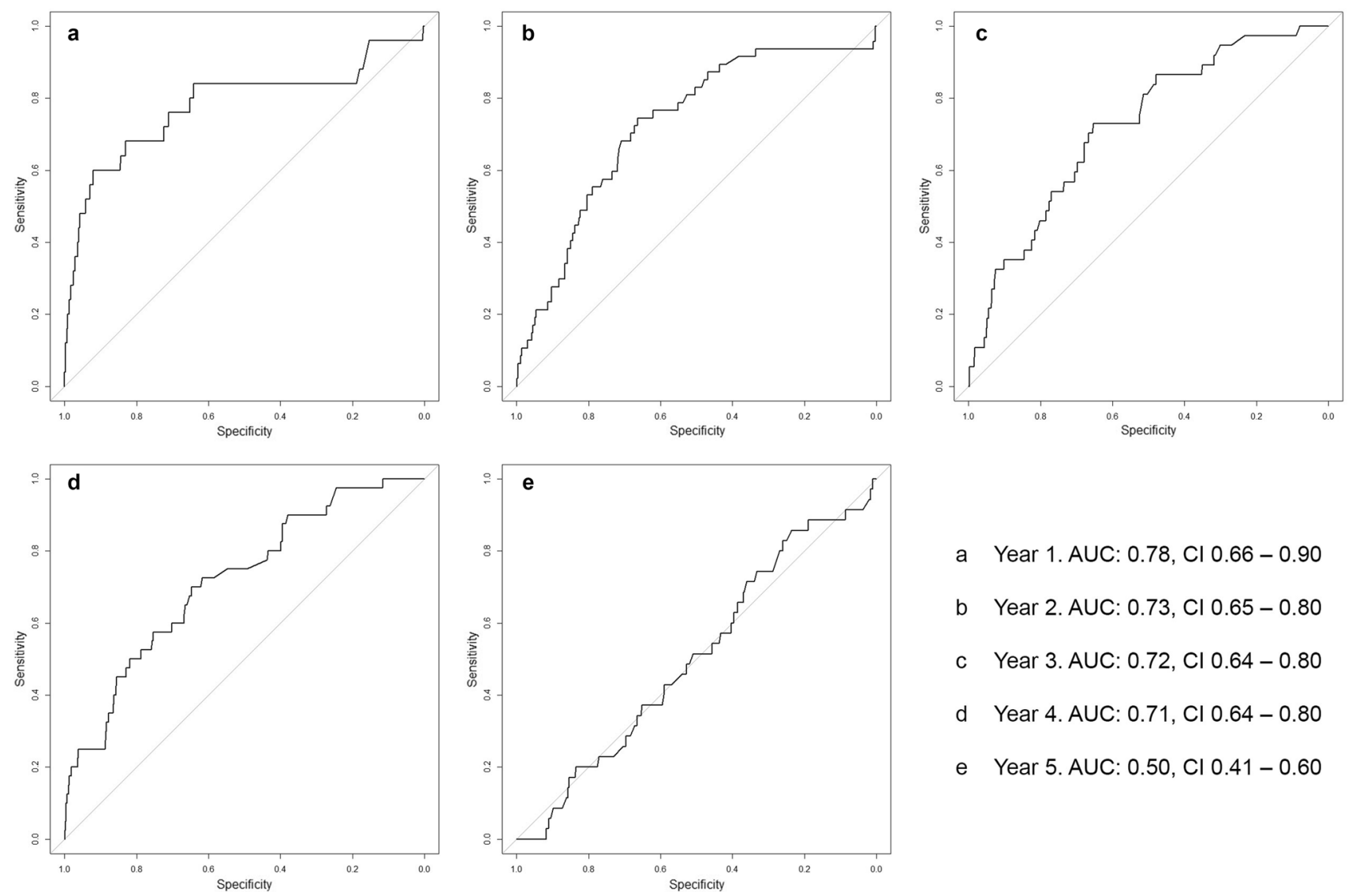

Fig. 4 Annual LRR-risk: ROC curves of the validation-cohort

Table 2 Evaluation of discriminative ability by subgroups

\begin{tabular}{lll}
\hline Subgroups & $\begin{array}{l}\text { AUC } \\
\text { (C-statistic) }\end{array}$ & CI (lower-upper) \\
\hline Age category at diagnosis (years) & & \\
$\quad<50$ & 0.69 & $0.61-0.78$ \\
$50-59$ & 0.75 & $0.66-0.83$ \\
$60-69$ & 0.71 & $0.62-0.81$ \\
$\geq 70$ & 0.75 & $0.68-0.82$ \\
Type of surgery & & \\
Breast conserving & 0.72 & $0.67-0.78$ \\
$\quad$ Mastectomy & 0.72 & $0.65-0.78$ \\
Intrinsic biological subtype & & \\
Luminal A/B & 0.71 & $0.66-0.76$ \\
Her2neu positive & 0.60 & $0.43-0.76$ \\
Triple negative & 0.73 & $0.64-0.82$ \\
\hline
\end{tabular}

\section{Discussion}

An essential step towards the implementation of prediction tools in daily clinical practice is the validation in the target population (Steyerberg 2009). The present study is the first one testing the Dutch INFLUENCE-nomogram with external data from another country. Although its predictions for the LRR-risk in the German cohort comprising 6520 breast cancer patients were less accurate than in the Dutch modeling-cohort, it did not perform worse in terms of discrimination-ability (C-statistic/AUC German validation-cohort: 0.73, CI 0.69-0.77 vs. C-statistic/AUC Dutch modeling-cohort: 0.71, CI 0.69-0.73).

Germany and the Netherlands are direct European neighbors which have many things in common. Both the Netherlands Cancer Registry and the German Tumor Center Regensburg (as part of the Population based Cancer Registry Bavaria) are member of the European Network of Cancer Registries (https://www.encr.eu/) and follow the mandatory data-collection standards and dataset requirements developed by this network. But the similarities between the two countries go beyond registration rules. Similarities are also reflected by highly similar patient and tumor characteristics. 
Moreover, the national breast cancer treatment guidelines of the Netherlands and Germany exhibit a large degree of congruency, since they rest on the same evidence base like in many countries (NABON 2012; Leitlinienprogramm Onkologie (Deutsche Krebsgesellschaft, Deutsche Krebshilfe, AWMF) 2019; Wolters et al. 2012). Nevertheless, some substantial differences concerning treatment modalities can be observed, pointing to different national preferences in breast cancer treatment. As a matter of fact, the breastconserving surgery rate in the Dutch cohort is $21.5 \%$ lower than in the German cohort, which also might explain the less frequent use of adjuvant radiotherapies. There are several potential reasons for this difference. First of all, the Dutch cohort derives from the years 2003 to 2006, whereas half of the German patients were treated thereafter. Between 2000 and 2012, the rate of breast-conserving surgery in the Netherlands progressed from 54 to $72 \%$ (Maaren et al. 2018). Second, one has to bear in mind that the Dutch patients as a whole are compared to a single region in Germany. Even in a small country like the Netherlands, large interregional variation exists concerning the use of breast-conserving surgery. According to a recent publication of van Maaren et al. (Maaren et al. 2018), some Dutch regions featured breastconserving surgery rates slightly below $80 \%$ already in the last decade, while others did not reach the $60 \%$ threshold as late as 2015 . Variation only decreased slightly after adjusting for different case mixes. It is very likely that a similar variation can be observed in Germany. The hospitals in the southern German region that we used for validation very actively participate in scientific research, which explains their early and broad implementation of the breast-conserving approach. However, the different national preferences concerning the surgical approach should not have influenced the results of our study, since the LRR-rate is comparable between breast-conserving surgery and mastectomy (Yang et al. 2008). Moreover, type of surgery does not contribute directly to the predictions of the INFLUENCE-nomogram, since breast-conserving surgery was strongly related to radiation therapy and, therefore, only the latter variable was included in the model (Witteveen et al. 2015).

For the considerably lower rate of endocrine therapy in the Netherlands, there might be another explication. The hormone status was unknown for over $20 \%$ of the Dutch patients, presumably because no tests were performed. Consequently, these patients were not eligible for hormone therapy. However, still only two-thirds of the patients with known hormone status received hormone therapy compared to around $90 \%$ in the German cohort.

Regardless of such differences, the LRR-rate was comparable between both countries and it seems justified to use the German cohort for external validation. Even if therapyallocation in both cohorts is different to a certain degree, the same surgical techniques, drugs for hormonal- and chemotherapy and radiation-schemes are used (NABON 2012; Leitlinienprogramm Onkologie (Deutsche Krebsgesellschaft, Deutsche Krebshilfe, AWMF) 2019). With a total of 184 recurrence events, it also meets an important formal requirement for an external validation, as according to Vergouwe et al. at least 100 events and 100 "nonevents" are necessary to determine whether a prediction tool performs well or not (Vergouwe et al. 2005). The rate of $2.8 \%$ LRR in the German validation-cohort is mildly, but not significantly $(p=0.205)$ above the level in the Dutch modelingcohort (2.6\%). Recently, van Maaren et al. published a paper reviewing long-term recurrence rates for breast cancer based on comprehensive NCR data from 2005 showing the hazard on LRR-events of Her2neu-positive and triple-negative patients peaks within the second post-surgical year and drops thereafter (Maaren et al. 2018). No clear trends were seen in Luminal A or B patients. The findings concerning the three latter groups could be confirmed within the German validation-cohort. No clear trend was to be seen with the Her2neu-positive patients. One reason for that might be the small number of patients within this group, which is also reflected by large confidence intervals-one recurrence event more or less can already change the situation considerably. Another possible reason for these differing observations are new developments in therapy. After the introduction of antibody therapy around 2005, Her2neu-positive patients were increasingly treated with Trastuzumab, which positively influences the outcome. Some of the patients in the German validation-cohort received this kind of therapy, while others did not. Obviously, no clear trends for this subgroup can be deducted from analyzing such a heterogeneous sub-population.

The INFLUENCE-tool's accuracy in the validationcohort was poor according to the Hosmer-Lemeshow test. A fact, which must not be overrated. Of course, the $p$ value is considerably lower than 0.05 , which is commonly regarded as a reasonable threshold between good and poor accuracy. The discrepancy between predicted and observed values partly may be attributed to the large confidence intervals caused by the relatively small number of events in the German validation-cohort. However, even if this aspect is taken into account, one can see that observed and predicted values do not differ by mere coincidence. Actually, the INFLUENCE-algorithm systematically underestimates the actual risk in each of the risk-stratified quintiles. A reason for that might be that the LRR-rate in the German cohort is slightly_-but not significantly_higher than in the Dutch modeling-cohort, while generally more adjuvant radio-, chemo, and endocrine therapies (which the INFLUENCEnomogram associates with a lower LRR-risk) are performed. This could possibly reflect moderate differences in therapy perception between the two populations, which could be an interesting topic for further investigation. 
For clinical use, accuracy is less important than discriminative ability, anyway. Health professionals seek to know whether their patients require intensified follow-up, because early detection of recurrence events is associated with superior outcomes (Lu et al. 2009; Sangen et al. 2013; Schneble et al. 2014). On the other hand, it is desirable to spare lowrisk patients the psychological and the health care system as such the financial burden of overly intensive follow-up schemes (Puglisi et al. 2014). To develop personalized follow-up pathways, physicians most probably will use the INFLUENCE-nomogram together with some kind of cutoff. The ROC curve depicts sensitivity and specificity for every possible threshold which can be used with the INFLUENCE tool. The C-statistic/AUC, therefore, represents the discriminative ability of the algorithm. For the 5-year overall LRRrisk algorithm, the C-statistic/AUC was 0.71 in the Dutch modeling-cohort; almost the same value was obtained by the first external validation with another Dutch cohort from 2007 and 2008. With the German patients analyzed within this study, the C-statistic/AUC was even slightly larger (0.73); this indicates good external validity. The number 0.73 means that if a-from the statistical point of view-ideal threshold of $1.6 \%$ was chosen, more than $70 \%$ of the high risk and more than $65 \%$ of the low-risk patients would be classified correctly, which, if implemented in daily clinical practice, would be an important step towards personalized medicine. The prediction tool also turned out to be robust against differences in population features, as no decline in model performance could be seen in any of the age-, type of surgery-, and intrinsic biological subtype-stratified subgroup analyses, except Her2neu-positive patients. While this also may be attributed to the issues with this special subgroup discussed earlier, the re-evaluation of Her2neu as an independent predictor in the INFLUENCE-model should be considered. According to Witteveen et al. the implementation of Her2neu did not improve the performance of the INFLUENCE-nomogram and consequently was omitted. However, the algorithm is based on patients from 2003 to 2006, which, as previously mentioned, was a period of change, as far as Her2neu is concerned and nowadays it is believed to have considerable influence on the outcome of interest (Gamucci et al. 2013; McGuire et al. 2017).

Focusing on the time-dependent models, discriminationability shows a negative gradient. The $\mathrm{C}$-statistic/AUC moderately decreases mildly from year 1 to 4 ; in year 5 it suddenly drops to 0.50 , indicating that there is no discriminative ability left. Notably, this is not a random phenomenon to be observed only in the validation-cohort. Internal validation based on the modeling-cohort returned a $\mathrm{C}$-statistic/AUC of 0.84 for the first year and constantly declined until year five to a $\mathrm{C}$-statistic/AUC of 0.62 . While it is not surprising that the model performance is better in the modeling than in the validation-cohort, it must be stated though, that the
INFLUENCE-nomogram obviously has some difficulties in predicting late recurrence events. Maybe this issue could be solved by updating the INFLUENCE-tool on a more recent modeling-cohort and re-evaluating the set of influence variables, like proposed above. It has to be acknowledged though that the occurrence of LRR could be influenced by unknown confounders, which might impede substantial improvement of model performance (Meads et al. 2012).

\section{Conclusion}

The INFLUENCE-nomogram can effectively assist health professionals in determining primarily cured breast cancer patients' individualized risk for locoregional recurrence. Remarkably, its overall prognostic ability is close to equal when used either in the German validation or the Dutch modeling-cohort, thus underlining international generalizability. Future research should aim to incorporate other important influencing factors to further enhance timedependent performance.

Funding Funded by the Deutsche Forschungsgemeinschaft (DFG, German Research Foundation)-project number 417891978.

\section{Compliance with ethical standards}

Conflict of interest V. Voelkel, T. Draeger, C. Groothuis-Oudshoorn, L. de Munck, T. Hueting, M. Gerken, M. Klinkhammer-Schalke, M. Lavric, and S. Siesling declare that they have no conflicts of interest or financial ties to disclose.

Research involving human and animal participants This article does not contain any studies with human participants or animals performed by any of the authors.

Informed consent Informed consent is given by patients and obtained by the doctor or clinician prior to reporting data to the cancer registries.

Open Access This article is distributed under the terms of the Creative Commons Attribution 4.0 International License (http://creativeco mmons.org/licenses/by/4.0/), which permits unrestricted use, distribution, and reproduction in any medium, provided you give appropriate credit to the original author(s) and the source, provide a link to the Creative Commons license, and indicate if changes were made.

\section{References}

Bamber D (1975) The area above the ordinal dominance graph and the area below the receiver operating characteristic graph. J Math Psychol 12(4):387-415. https://doi.org/10.1016/0022-2496(75)90001 $-2$

Bayerisches Landesamt für Statistik und Datenverarbeitung (2014) Zensus 2011: Daten zur Altersstruktur der Bevölkerung für den Regierungsbezirk Oberpfalz, München 
Clopper CJ, Pearson ES (1934) The use of confidence or fiducial limits illustrated in the case of the binOMIAL. Biometrika 26(4):404413. https://doi.org/10.1093/biomet/26.4.404

DeLong ER, DeLong DM, Clarke-Pearson DL (1988) Comparing the areas under two or more correlated receiver operating characteristic curves: a nonparametric approach. Biometrics 44(3):837-845

Deutsches Institut für Medizinische Dokumentation und Information (2017) ICD-10-GM version 2017 systematisches verzeichnis internationale statistische klassifikation der krankheiten und verwandter gesundheitsprobleme, 10. Revision-German Modification. https://www.icd-code.de/ops/code/OPS.html. Accessed 19 Nov 2017

Deutsches Institut für Medizinische Dokumentation und Information (2019) OPS-klassifikation. https://www.dimdi.de/static/de/klass i/ops/index.htm. Accessed 15 Feb 2018

Gamucci T, Vaccaro A, Ciancola F et al (2013) Recurrence risk in small, node-negative, early breast cancer: a multicenter retrospective analysis. J Cancer Res Clin Oncol 139(5):853-860. https:// doi.org/10.1007/s00432-013-1388-2

Geurts YM, Witteveen A, Bretveld R et al (2017) Patterns and predictors of first and subsequent recurrence in women with early breast cancer. Breast Cancer Res Treat 165(3):709-720. https:// doi.org/10.1007/s10549-017-4340-3

Goldhirsch A, Wood WC, Coates AS et al (2011) Strategies for subtypes-dealing with the diversity of breast cancer: highlights of the St Gallen international expert consensus on the primary therapy of early breast cancer 2011. Ann Oncol 22(8):1736-1747. https://doi.org/10.1093/annonc/mdr304

Hermanek P, Wittekind C (1994) Residual tumor (R) classification and prognosis. Semin Surg Oncol 10(1):12-20

Holleczek B, Arndt V, Stegmaier C et al (2011) Trends in breast cancer survival in Germany from 1976 to 2008-a period analysis by age and stage. Cancer Epidemiol 35(5):399-406. https://doi. org/10.1016/j.canep.2011.01.008

Holleczek B, Brenner H (2012) Trends of population-based breast cancer survival in Germany and the US: decreasing discrepancies, but persistent survival gap of elderly patients in Germany. BMC Cancer 12:317. https://doi.org/10.1186/1471-2407-12-317

Hosmer DW Jr, Lemeshow S, Sturdivant RX (2013) Applied Logistic Regression. Wiley, Hoboken

Kundu S, Aulchenko YS, van Duijn CM et al (2011) PredictABEL: an $\mathrm{R}$ package for the assessment of risk prediction models. Eur J Epidemiol 26(4):261-264. https://doi.org/10.1007/s1065 4-011-9567-4

Leitlinienprogramm onkologie (deutsche krebsgesellschaft, deutsche krebshilfe, AWMF) (2019) S3-leitlinie früherkennung, diagnose, therapie und nachsorge des mammakarzinoms, version 4.1, 2018 AWMF registernummer: 032-045OL, https://www.leitlinienprogr amm-onkologie.de/leitlinien/mammakarzinom/(abgerufen am 05.12.2018)

Lu WL, Jansen L, Post WJ et al (2009) Impact on survival of early detection of isolated breast recurrences after the primary treatment for breast cancer: a meta-analysis. Breast Cancer Res Treat 114(3):403-412. https://doi.org/10.1007/s10549-008-0023-4

McGuire A, Lowery AJ, Kell MR et al (2017) Locoregional recurrence following breast cancer surgery in the trastuzumab era: a systematic review by subtype. Ann Surg Oncol 24(11):3124-3132. https ://doi.org/10.1245/s10434-017-6021-1

Meads C, Ahmed I, Riley RD (2012) A systematic review of breast cancer incidence risk prediction models with meta-analysis of their performance. Breast Cancer Res Treat 132(2):365-377. https ://doi.org/10.1007/s10549-011-1818-2

NABON (2012) Breast cancer, dutch guideline, version 2.0. https:// www.oncoline.nl/mammacarcinoom

Puglisi F, Fontanella C, Numico G et al (2014) Follow-up of patients with early breast cancer: is it time to rewrite the story? Crit Rev
Oncol Hematol 91(2):130-141. https://doi.org/10.1016/j.critr evonc.2014.03.001

Robin X, Turck N, Hainard A et al (2011) pROC: an open-source package for $\mathrm{R}$ and $\mathrm{S}+$ to analyze and compare ROC curves. BMC Bioinformatics 12:77. https://doi.org/10.1186/1471-2105-12-77

Schemper M, Smith TL (1996) A note on quantifying follow-up in studies of failure time. Control Clin Trials 17(4):343-346

Schneble EJ, Graham LJ, Shupe MP et al (2014) Current approaches and challenges in early detection of breast cancer recurrence. J Cancer 5(4):281-290. https://doi.org/10.7150/jca.8016

Stewart BW, Wild CP (2014) World Cancer Report 2014. Online-Ausg. EBL-Schweitzer, International Agency for Research on Cancer/ World Health Organization, Lyon

Steyerberg EW, Vickers AJ, Cook NR et al (2010) Assessing the performance of prediction models: a framework for traditional and novel measures. Epidemiology 21(1):128-138. https://doi. org/10.1097/EDE.0b013e3181c30fb2

Steyerberg EW (2009) Clinical prediction models: a practical approach to development, validation, and updating. statistics for biology and health. Springer, New York

Tumorzentrum Regensburg (2019) https://www.tumorzentrum-regen sburg.de/tumorzentrum-regensburg.html. Accessed 15 Feb 2018

van der Sangen MJC, Poortmans PMP, Scheepers SWM et al (2013) Prognosis following local recurrence after breast conserving treatment in young women with early breast cancer. Eur J Surg Oncol 39(8):892-898. https://doi.org/10.1016/j.ejso.2013.05.004

van Maaren MC, Strobbe LJA, Koppert LB et al (2018) Nationwide population-based study of trends and regional variation in breastconserving treatment for breast cancer. Br J Surg 105(13):17681777. https://doi.org/10.1002/bjs.10951

van Maaren MC, de Munck L, Strobbe LJ et al (2018) 10-year recurrence rates for breast cancer subtypes in the Netherlands: A large population-based study. Int J Cancer. 1:1. https://doi.org/10.1002/ ijc.31914

Vergouwe Y, Steyerberg EW, Eijkemans MJC et al (2005) Substantial effective sample sizes were required for external validation studies of predictive logistic regression models. J Clin Epidemiol 58(5):475-483. https://doi.org/10.1016/j.jclinepi.2004.06.017

von Elm E, Altman DG, Egger M et al (2007) The strengthening the reporting of observational studies in epidemiology (STROBE) statement: guidelines for reporting observational studies. The Lancet 370(9596):1453-1457. https://doi.org/10.1016/S0140 -6736(07)61602-X

Witteveen A, Vliegen IMH, Sonke GS et al (2015) Personalisation of breast cancer follow-up: a time-dependent prognostic nomogram for the estimation of annual risk of locoregional recurrence in early breast cancer patients. Breast Cancer Res Treat 152(3):627636. https://doi.org/10.1007/s10549-015-3490-4

Witteveen A (2015) Nomogram-influence project: time-dependent prognostic nomogram for the estimation of the 5 year and conditional annual risk of locoregional recurrence in early breast cancer patients. https://www.utwente.nl/en/techmed/influence/nomog $\mathrm{ram} /$. Accessed 28 Nov 2018

Witteveen A Influence (2019) Five-year locoregional recurrence risk in breast cancer patients. https://www.evidencio.com/models/ show/562. Accessed 28 Nov 2018

Wolters R, Regierer AC, Schwentner L et al (2012) A comparison of international breast cancer guidelines - do the national guidelines differ in treatment recommendations? Eur J Cancer 48(1):1-11. https://doi.org/10.1016/j.ejca.2011.06.020

Yang SH, Yang KH, Li YP et al (2008) Breast conservation therapy for stage I or stage II breast cancer: a meta-analysis of randomized controlled trials. Ann Oncol 19(6):1039-1044. https:// doi.org/10.1093/annonc/mdm573

Yoshimura A, Ito H, Nishino Y et al (2018) Recent Improvement in the Long-term Survival of Breast Cancer Patients by Age and Stage 
in Japan. J Epidemiol 28(10):420-427. https://doi.org/10.2188/ jea.JE20170103

Youden WJ (1950) Index for rating diagnostic tests. Cancer 3(1):32-35. https://doi.org/10.1002/1097-0142(1950)3:1\%3c32:AID-CNCR2 $2 \% 3 \mathrm{e} 3.0 . \mathrm{CO} ; 2-3$

Youngstrom EA (2013) A primer on receiver operating characteristic analysis and diagnostic efficiency statistics for pediatric psychology: we are ready to ROC. J Pediatr Psychol 39(2):204221. https://doi.org/10.1093/jpepsy/jst062

Publisher's Note Springer Nature remains neutral with regard to jurisdictional claims in published maps and institutional affiliations. 